\title{
Plastic Strain Arrangement in Copper Single Crystals in Sliding
}

\author{
Andrey V. Chumaevskii, 2, a), Sergei Yu. Tarasov ${ }^{3,4, b)}$, \\ and Dmitry V. Lychagin ${ }^{1,2, c)}$ \\ ${ }^{1}$ National Research Tomsk State University, Tomsk, 634050, Russia \\ ${ }^{2}$ Tomsk State University of Architecture and Building, Tomsk, 634003, Russia \\ ${ }^{3}$ National Research Tomsk Polytechnic University, Tomsk, 634050, Russia \\ ${ }^{4}$ Institute of Strength Physics and Materials Science SB RAS, Tomsk, 634055, Russia

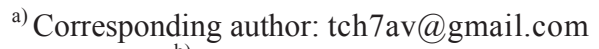 \\ b) tsy@ispms.tsc.ru \\ c)dvl-tomsk@mail.ru
}

\begin{abstract}
Deformation of tribologically loaded contact zone is one of the wear mechanisms in spite of the fact that no mass loss may occur during this process. Generation of optimal crystallographic orientations of the grains in a polycrystalline materials (texturing) may cause hardening and reducing the deformation wear. To reveal the orientation dependence of an individual gain and simplify the task we use copper single crystals with the orientations of the compression axis along [1 $\overline{1} \overline{1}]$ and [110]. The plastic deformation was investigated by means of optical, scanning electron microscopy and EBSD techniques. It was established that at least four different zones were generated in the course of sliding test, such as non-deformed base metal, plastic deformation layer sliding, crystalline lattice reorientation layer and subsurface grain structure layer. The maximum plastic strain penetration depth was observed on [110]-single crystals. The minimum stability of $[1 \overline{1} \overline{1}]$-crystals with respect to rotation deformation mode as well as activation of shear in the sliding contact plane provide for rotation deformation localization below the worn surface. The high-rate accumulation of misorientations and less strain penetration depth was observed on $[1 \overline{1} \overline{1}]$-crystals as compared to those of [110]-oriented ones.
\end{abstract}

Keywords: friction, single crystal, copper, fragmentation, EBSD, sliding

\section{INTRODUCTION}

Investigations into friction force-induced plastic deformation have become more and more sophisticated; at the same time more and more inhomogeneities are revealed in the course of it [1-5]. Such a situation is provided, first of all, by the presence of several loading components, friction-induced heat and cyclic character of loading stemming from alternating compressive and tensile shear stresses in the real contact areas. The use of single crystals for studying the specificity of deformation as depended on their orientations allows extending the knowledge obtained on the polycrystalline materials. It is known $[6,7]$ that various deformation-induced relief components are observed on the faces of both [110]- and [1 $\overline{1} \overline{1}]$-single crystalline samples of nickel, aluminum and copper under compression uniaxial loading. Along with that, these crystals reveal different propensity to the crystalline lattice reorientation. One of the reasons behind higher propensity of the [1 $\overline{1} \overline{1}]$-crystals to reorientation may be constraint shear conditions in the direction of free crystal's faces as well as accelerated accumulation of the same sign excess dislocation density in the bulk of the crystals. These specificities must have their effect on the deformation behavior of single crystalline samples under sliding test. It defines the choice of single crystal orientations in this work. Since copper is appropriate and widely used material model for tribological studies, it was chosen for the research.

International Conference on Physical Mesomechanics of Multilevel Systems 2014

AIP Conf. Proc. 1623, 91-94 (2014); doi: 10.1063/1.4898890

(C) 2014 AIP Publishing LLC 978-0-7354-1260-6/\$30.00 
Therefore, the objective of this work is to study the deformation surface relief formed on the copper crystal' lateral faces as well as structural segmentation and reorientation patterns using optical, scanning electron microscopy and EBSD techniques.

\section{MATERIAL AND METHODS}

Copper $3.5 \times 3.5 \times 7 \mathrm{~mm}$ commercial grade purity single crystals, grown according to Bridgeman method and cut out to have their compression axis orientation [110] or [1 $\overline{1} 1]$ were subjected to sliding tests with different friction force directions (Fig. 1). Sliding tests were carried out using a pin-on-disk tribometer «TRIBOtechnic». Sliding speed was $50 \mathrm{~mm} / \mathrm{s}$ at normal load $12 \mathrm{~N}$. The sliding path length was $200 \mathrm{~m}$. The counter body was made of quartz glass. The surface deformation-induced relief was examined using optical microscope LeicaDM 2500P, SEM instrument Tescan Vega II LMU attached with EBSD instrument.

\section{RESULTS AND DISCUSSION}

Friction-force-induced plastic deformation in solids may achieve very high strain, which is expressed in subsurface structural fragmentation (segmentation) and microcrystalline layer generation, crystalline lattice reorientation and shear by slip planes [4]. In view that all these processes occur simultaneously at different scales, we finally obtain different deformation stages below the worn surface. The complex of deformation zones, subjects to different deformation modes, defines the plastic deformation arrangement.

\section{Subsurface Plastic Deformation}

Plastic deformation in the subsurface of single crystals in sliding test occurs by shear in the close-packed slip planes so that shear traces show up on the crystal faces thus forming a surface deformation-induced relief. The SEM images of the relief, formed on the lateral sides of crystals with orientations in Fig. 1, are shown in Fig. 2. The deformed zone depth below the worn surface is 2 and $0.7 \mathrm{~mm}$ for [110]- and [1 $\overline{1} \overline{1}]$-single crystals, respectively. In addition, there is an essential difference in shear trace distribution over the deformed zone area between the crystals. The most part of the area in [110]-single crystal subsurface zone is occupied by intersecting slip bands belonging to two or even three slip systems. For [1 $\overline{1} \overline{1}]$-single crystals the area, occupied by the intersecting slip bands, is less by a factor of 3-5. The maximum slip band density is observed in the vicinity of the worn surface thus being reduced with the distance below it. Reoriented crystalline lattice layer and grain-subgrain structure layer are even closer to the worn surface.

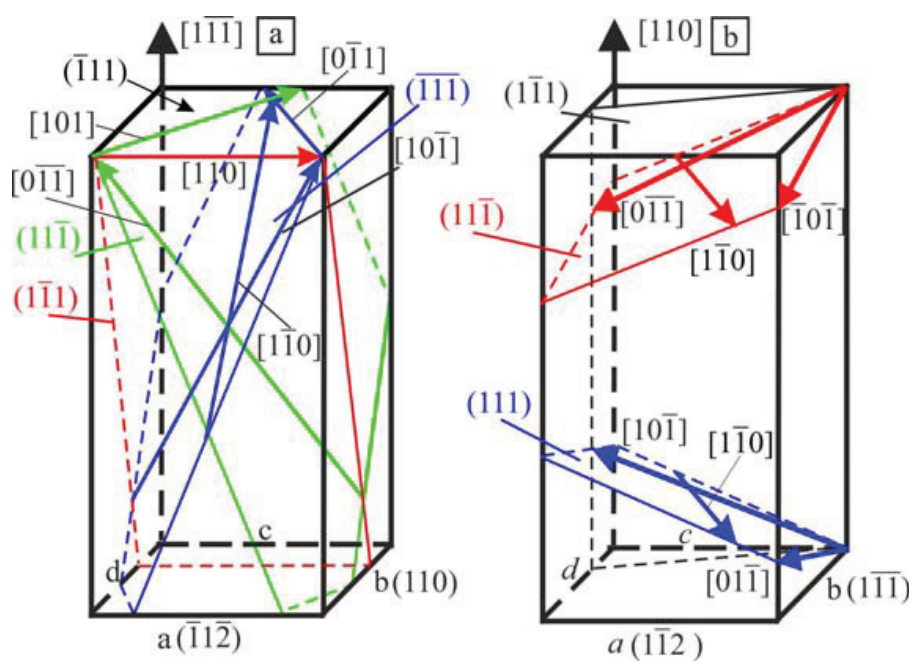

FIGURE 1. Crystallographic orientations of copper single crystals under the study 


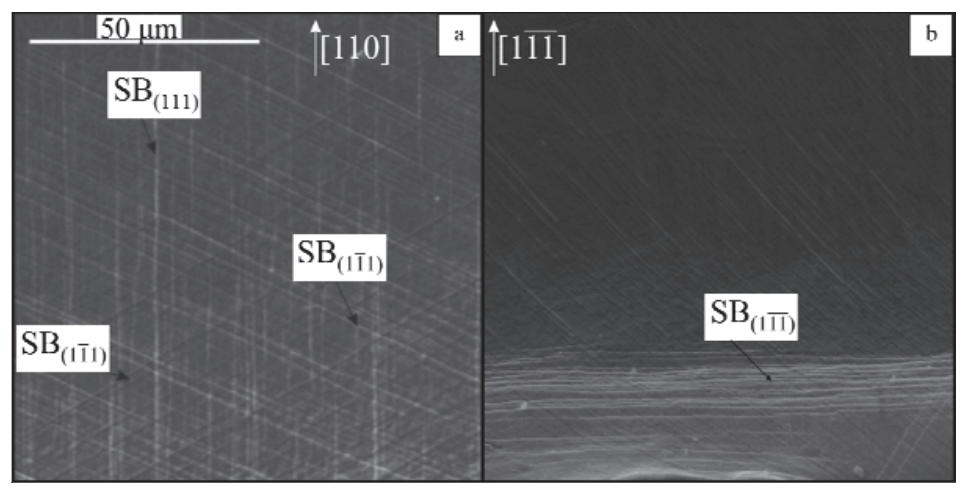

FIGURE 2. Deformation-induced relied on the lateral faces of the copper single crystals after sliding test

\section{Subsurface Crystalline Lattice Reorientation and Structural Segmentation}

The EBSD mapping of the crystalline lattice reorientation in the subsurface of single crystals after sliding is shown in Fig. 3. Three different reorientation areas designated as $A, B$, and $C$ are distinguished. The reorientation in $A$ and $B$ areas occurs by rotating the compression axis from initial $\langle 110\rangle$ to coincide with $\langle 112\rangle$ and $\langle 001\rangle$ respectively. The direction of sliding rotates from $\langle 111\rangle$ to $\langle 110\rangle$ are coincided in both cases. The perpendicular direction $\langle 112\rangle$ of the $B$-area rotates to coincide with $\langle 110\rangle$ while it stays unchanged for the $A$-area. The $A$-area experiences a rotation around $\langle 112\rangle$ while the crystalline lattice rotation in the $B$-area is more complex since all three axes change their orientations. The misorientations in the $C$-area are smooth and axis reorientation is similar to that found for the $B$-area. Therefore, these areas are characterized by crystalline lattice rotation and may be referred to as the crystalline lattice reorientation layer. The layer, where misorientation is accumulated enough to form grains and subgrains, is found closer to the worn surface.

The reorientation EBSD mapping for [1 $\overline{1} \overline{1}]$-single crystal is shown in Fig. 4 where one can see in the $A$-area compression axis, sliding direction and perpendicular to the sliding direction are changed to coincide with $\langle 110\rangle$, $\langle 112\rangle$, and $\langle 111\rangle$ respectively. In addition, there are some blurring around these orientations. For the $B$-area compression axis is changed to coincide with $\langle 001\rangle$ and $\langle 112\rangle$, while sliding axis to $\langle 110\rangle$. The [1 $\overline{1} \overline{1}]$-single crystal reoriented and deformed layer thicknesses are reduced as compared to those of [110]-ones. Such a result could be explained by low rotation stability of the compression axis as well as by feasibility of shear in the sliding contact plane.

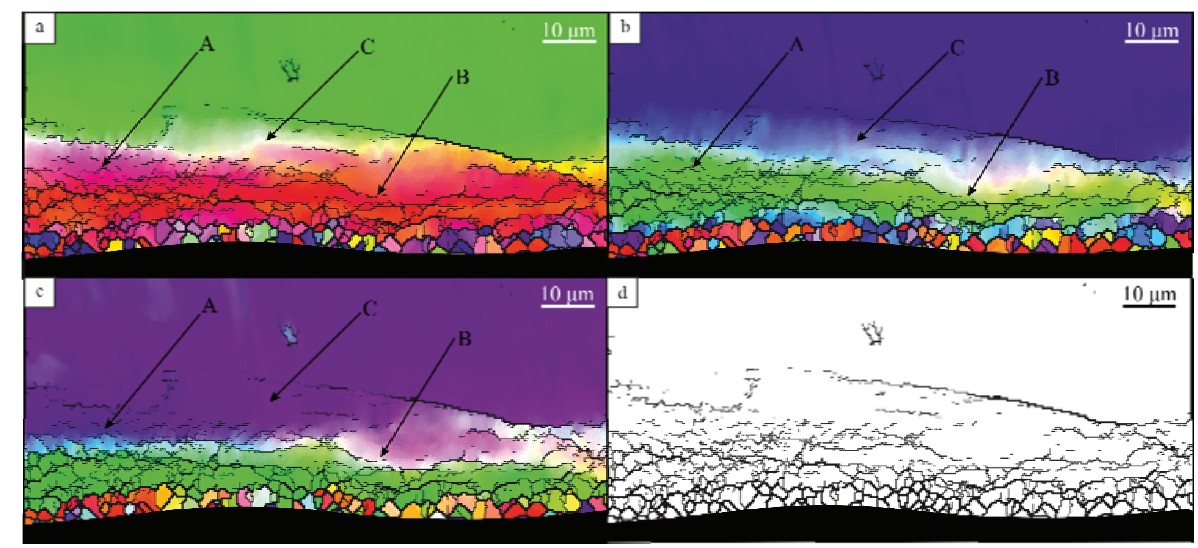

FIGURE 3. The EBSD mapping of the [110]-sample crystalline lattice reorientation for compression axis (a), sliding direction (b) and perpendicular to the sliding direction (c), grain boundary structure (d) 


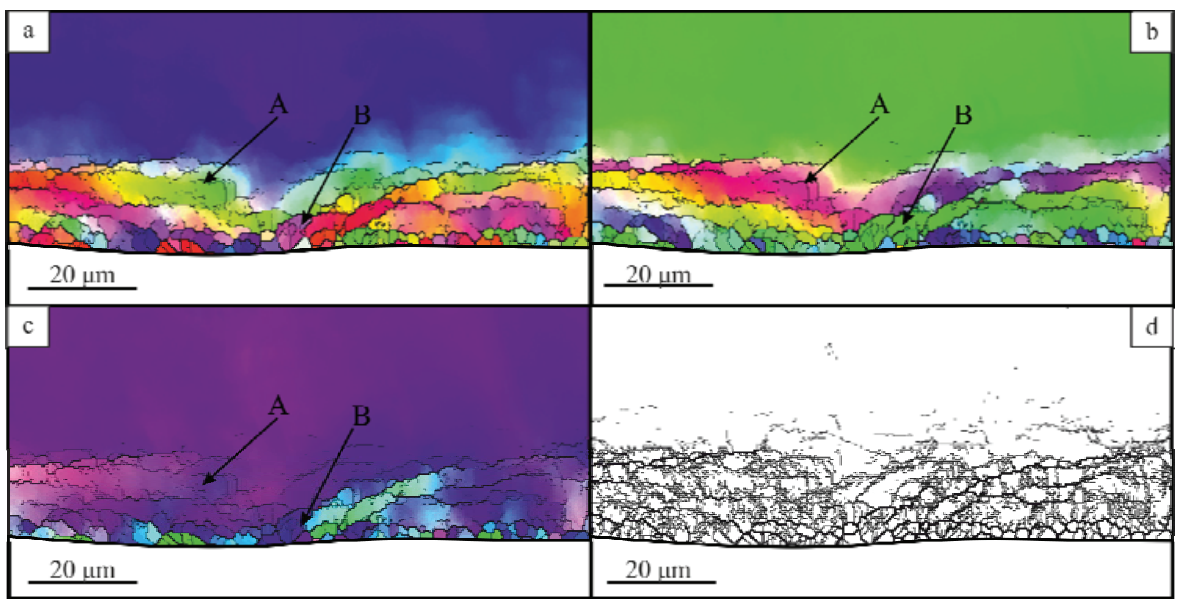

FIGURE 4. The EBSD mapping of the $[1 \overline{1} \overline{1}]$-sample crystalline lattice reorientation for compression axis (a), sliding direction (b), and perpendicular to the sliding direction (c), grain boundary structure (d)

Both for $[1 \overline{1} \overline{1}]$ - and [110]-single crystals in sliding the subsurface crystalline lattice rotation occurs preferably around the axis lying in the contact plane and being perpendicular to the sliding direction.

\section{CONCLUSION}

The results obtained in this work demonstrate the specificity of plastic deformation occurrence in the subsurface of single crystals as depended on normal and friction force directions. The friction-induced deformation generates at least four subsurface zones (layers) of different strain level accumulated as follows: fragmented grain-subgrain structure zone, reoriented crystalline structure zone, plastic deformation zone and finally non-deformed base lattice. The thickness of the deformed layer generated by sliding in [110]-single crystal is more than that of the [1 $\overline{1} \overline{1}]$ single crystal. The same situation is with the thicknesses of both reoriented structure and grain-subgrain layers. It was explored that wear particle detachment is easier in [1 $\overline{1} \overline{1}]$-single crystal in view that the sliding contact plane is simultaneously the close-packed slip plane too. This fact may explain the existence of less strain propagation depth in this crystal. In addition, it should be noted that [1 $\overline{1} \overline{1}]$-single crystal possesses lower rotation stability so that subsurface strain localization becomes more feasible thus having a reducing effect on the strain propagation depth. A standalone fact is that the crystalline lattice rotation in the reoriented zones is most favorable with respect to axis perpendicular to the friction force direction and lying in the sliding contact plane. So the orientation dependence of the plastic deformation at different scale levels in copper single crystal was demonstrated.

\section{ACKNOWLEDGEMENT}

The work is supported by partial support of Tomsk State University Development Program (obtaining the samples and characterization) and RFBR grant No. 13-08-00324 (tribological testing). The authors are grateful to the "Analytic Center for Geochemistry of Natural Systems" for using the analytic equipment.

\section{REFERENCES}

1. S. Tarasov, V. Rubtsov, and A. Kolubaev, Wear 268, 59 (2010).

2. Y. Ohno, J. Inotani, Y. Kaneko, and S. Hashimoto, J. Japan Inst. Metals 74(6), 384 (2010).

3. A. Emge, S. Karthikeyan, and D. A. Rigney, Wear 267, 562 (2009).

4. S. Yu. Tarasov, D. V. Lychagin, and A. V. Chumaevski, Appl. Surf. Sci. C 274, 22 (2013).

5. K. L. Johnson, Contact Mechanics (University Press, Cambridge, 1999).

6. D. V. Lychagin, Phys. Mesomech. 9(3-4), 95 (2006).

7. D. V. Lychagin, V. A. Starenchenko, and Yu. V. Solov'eva, Phys. Mesomech 9(1-2), 63 (2006). 